\title{
Cholangiocarcinoma: An Emerging Target for Molecular Therapy
}

\author{
Ilya Tsimafeyeu ${ }^{a}$ Mark Temper $^{\text {b }}$

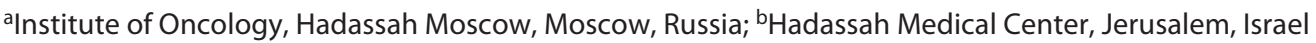

\section{Key Messages}

- Cholangiocarcinoma has been traditionally considered a tumor with poor prognosis.

- Only $35 \%$ of patients are candidates for surgical treatment, of which another $35 \%$ subsequently relapse within 2 years.

- Overall survival of metastatic cholangiocarcinoma patients is $<1$ year.

- Novel therapies with fibroblast growth factor receptor, isocitrate dehydrogenase, and checkpoint inhibitors gave new hope in treatment of advanced tumors.

\section{Keywords}

Cholangiocarcinoma - Metastatic disease $\cdot$ Fibroblast growth factor receptors $2 \cdot$ Isocitrate dehydrogenase . Targeted therapy $\cdot$ Immunotherapy

\begin{abstract}
Background: Cholangiocarcinoma has been traditionally considered a tumor with poor prognosis. Until now, surgical treatment has been the only more or less effective approach. Summary: Over 10 years, chemotherapy with a combination of gemcitabine and cisplatin remains the standard first-line therapy for patients with locally advanced or metastatic cholangiocarcinoma, which leads to a median overall survival of 11.7 months. Several inhibitors of HER (ERBB), HGF/cMET, Hedgehog, KRAS-BRAF-MEK-ERK, and PI3K/AKT/mTOR signaling pathways did not show their superiority to standard chemotherapy. The rise of hope is associated with the emergence of novel fibroblast growth factor receptors and isocitrate dehydrogenase inhibitors as well as immune checkpoint inhibitors.

(c) 2021 The Author(s)

Published by S. Karger AG, Basel
\end{abstract}

\section{Introduction}

Biliary tract cancers constitute a group of tumors arising from the epithelium of intra- and extrahepatic bile ducts (cholangiocarcinoma) and the gallbladder [1]. The term "cholangiocarcinoma" comprises 3 types of tumors with different risk factors, characteristics, and treatment approaches: these are intra- and extrahepatic tumors and malignancies involving the bile ducts of the hilar bifurcation, belonging to extrahepatic tumors (Klatskin tumor). Biliary tract cancers are thought to account for about 3\% of all gastrointestinal cancers; intrahepatic cholangiocarcinomas account for about $10 \%$ of cases of primary liver cancers. Among cholangiocarcinomas, Klatskin tumor accounts for $50 \%$ of cases, of which $40 \%$ are distal tumors and $10 \%$ are intrahepatic tumors [1]. The average incidence of cholangiocarcinoma in the United States is 1.26 cases per 100,000 population $[1,2]$.

Cholangiocarcinoma has been traditionally considered a tumor with poor prognosis. Until now, surgical treatment has been the only more or less effective approach. However, even it is a viable treatment option for
Correspondence to:

Ilya Tsimafeyeu, i.tsimafeyeu@ hadassah.moscow 
Table 1. Results of trials in patients with metastatic cholangiocarcinoma

\begin{tabular}{|c|c|c|c|}
\hline Study/result & $\begin{array}{l}\text { Objective } \\
\text { response rate, } \\
\%\end{array}$ & $\begin{array}{l}\text { Progression- } \\
\text { free survival, } \\
\text { months }\end{array}$ & $\begin{array}{l}\text { Overall } \\
\text { survival, } \\
\text { months }\end{array}$ \\
\hline \multicolumn{4}{|l|}{ MET inhibition } \\
\hline Phase 2 study, cabozantinib [12] & 0 & 1.8 & 5.2 \\
\hline \multicolumn{4}{|l|}{ FGFR inhibition } \\
\hline Phase 2 study (FIGHT-202), pemigatinib [19] & 35.5 & 6.9 & 21.1 \\
\hline Phase 2 study, infigratinib [21] & 26.9 & 6.8 & 12.5 \\
\hline \multicolumn{4}{|l|}{ IDH1 inhibition } \\
\hline Phase 3 study (ClarIDHy), ivosidenib [25, 26] & 2 & 2.7 & 10.3 \\
\hline Phase 1 study, ivosidenib [27] & 5 & 3.8 & - \\
\hline \multicolumn{4}{|l|}{ Immune checkpoint inhibitors } \\
\hline Phase 1 study, nivolumab with gemcitabine and cisplatin ${ }^{1}[30]$ & 37 & 4.2 & 15.4 \\
\hline Phase 2 study, nivolumab [31] & 22 & 3.68 & 14.24 \\
\hline Phase 1b study (KEYNOTE 028), pembrolizumab [32] & 13 & 1.8 & 6.2 \\
\hline Phase 2 study (KEYNOTE 158), pembrolizumab [32] & 5.8 & 2 & 7.4 \\
\hline \multicolumn{4}{|l|}{ Phase 1 study [33] } \\
\hline Durvalumab alone & 4.8 & - & 8.1 \\
\hline Durvalumab/tremelimumab & 11 & & 10.1 \\
\hline \multicolumn{4}{|l|}{ Phase 2 randomized study [34] } \\
\hline Atezolizumab alone & 32.3 & 1.87 & Not mature \\
\hline Atezolizumab/cobimetinib & 45.1 & 3.65 & \\
\hline
\end{tabular}

FGFR, fibroblast growth factor receptor; IDH, isocitrate dehydrogenase. ${ }^{1}$ This combination was studied in treatment-naive patients.

only $35 \%$ of patients [3], of which another $35 \%$ subsequently relapse within 2 years [4]. Over 10 years, chemotherapy with a combination of gemcitabine and cisplatin remains the standard first-line therapy for patients with locally advanced or metastatic cholangiocarcinoma, which leads to a median overall survival of 11.7 months [5]. Previously, upon disease progression on first-line chemotherapy, patients were left without effective treatment options. Attempts to use different chemotherapy regimens, both single-agent chemotherapy and combinations, have failed [6-8]. Fluoropyrimidines and their combinations with oxaliplatin or irinotecan are empirically prescribed as subsequent-line therapy in order not to leave the patient untreated [1].

In the era of targeted therapy, researchers tried to use the HER (ERBB), Hedgehog, KRAS-BRAF-MEK-ERK, and $\mathrm{PI} 3 \mathrm{~K} / \mathrm{AKT} / \mathrm{mTOR}$ signaling pathway inhibitors, which have proven effective in other tumors, in the treatment of biliary tract cancer; however, no study showed their superiority to standard chemotherapy [9]. Certain hopes were also associated with the inhibition of MET signaling, which is often activated in cholangiocarcinoma and promotes carcinogenesis by increasing angiogenesis and invasion. MET is overexpressed in $20-68 \%$ of chol- angiocarcinomas $[10,11]$. In the phase 2 study, previously treated patients with inoperable or metastatic cholangiocarcinoma received cabozantinib, a potent VEGFR and MET inhibitor [12]. Unfortunately, cabozantinib demonstrated limited activity and significant toxicity. The median progression-free survival was only 1.8 months and the median overall survival did not exceed 6 months. Again, targeted therapy has failed. Most recently, scientists have turned their attention to new molecular alterations in cholangiocarcinoma cells, namely, in the family of fibroblast growth factor and fibroblast growth factor receptors (FGFRs), as well as in isocitrate dehydrogenase (IDH) 1 and 2 (Table 1). Moreover, immunotherapy that has shown promising results in other tumors has turned to cholangiocarcinoma (Table 1).

\section{FGFR Inhibition}

FGFR alterations occur on average in $7.1 \%$ of all cancer patients [13] and are the potential therapeutic targets in some gastrointestinal tumors, for example, gastric cancer $[14,15]$. Cholangiocarcinoma is a tumor with the most common FGFR alterations. Thus, rearrangements 
and fusions in the FGFR genes, in particular, FGFR2, were detected in $6.1-16 \%$ of patients $[13,16,17]$. Therefore, it was logical to develop and study FGFR2 inhibitors in these patients.

On April 17, 2020, the US Food and Drug Administration (FDA) approved under accelerated process the first FGFR1-3 inhibitor, pemigatinib, for the treatment of patients with metastatic cholangiocarcinoma harboring FGFR2 gene fusions or rearrangements, previously treated with standard chemotherapy [18]. The FDA's decision was based on the results of a multicenter, open-label, phase 2 trial FIGHT-202 [19]. This study screened 1,206 patients, of which 107 patients had FGFR2 gene fusions or rearrangements, as detected using the FoundationOne $\mathrm{CDx}$ test. Pemigatinib was used at a dose of $13.5 \mathrm{mg}$ orally, daily for a 21 -day cycle (2 weeks on, 1 week off). The primary endpoint was the objective response rate in patients with FGFR2 alterations.

With a median follow-up of 17.8 months, the objective responses were reported in 38 patients, which amounted to $35.5 \%$. Three patients achieved complete response to treatment. This was in line with the statistical hypothesis and the study reached its primary endpoint. The response developed quite quickly, at 2.7 months, and was prolonged (taking into account the patient cohort and the type of tumor) - lasted for 7.5 months. The median progression-free survival was 6.9 months. The median overall survival with continued follow-up was calculated as 21.1 months. Hyperphosphatemia, a class-specific adverse event, was the most common all-grade toxicity with an incidence of $60 \%$. Sixty-four percent of patients developed grade $\geq 3$ adverse events: hypophosphatemia (12\%), arthralgia (6\%), stomatitis (5\%), hyponatremia (5\%), abdominal pain $(5 \%)$, and fatigue (5\%). Forty-five percent of patients experienced severe toxicity, with the most common events being abdominal pain (5\%), pyrexia (5\%), cholangitis (3\%), and pleural effusion (3\%). No deaths were considered treatment related.

Based on such results, one can undoubtedly agree with the FDA's decision to approve pemigatinib for the treatment of such an aggressive tumor, in which the survival of patients who did not respond to first-line therapy previously did not exceed 6.5-13.4 months. Pemigatinib increases this figure by at least 2 times. A phase 3 study FIGHT-302 has been announced and is ongoing [20]. It compares pemigatinib with the combination of gemcitabine and cisplatin as the first-line treatment for metastatic cholangiocarcinoma harboring FGFR2 gene rearrangements.

Targeted Therapy and Immunotherapy for Cholangiocarcinoma
The results of an efficacy study of the second FGFR1-3 inhibitor, infigratinib, were presented as a late-breaking abstract at the 2018 ESMO congress [21]. Patients with cholangiocarcinoma, resistant to standard chemotherapy, received oral infigratinib $125 \mathrm{mg}$ daily for 21 days of a 28-day cycle until unacceptable toxicity, disease progression, or consent withdrawal. The primary endpoint was the confirmed overall response rate as assessed by the investigators. The secondary endpoints included progression-free survival, disease control rate, overall survival, and safety.

The study enrolled 71 patients (62\% women; median age of 53 years) with FGFR2 fusion/translocation. The preliminary analysis showed the median treatment duration of 5.5 months and follow-up time of 8.4 months, with 62 patients having discontinued the treatment. The total objective response rate (confirmed and unconfirmed responses) was $31.0 \%$, with the rate of confirmed responses being $26.9 \%$. The rate was higher in patients who received $\leq 1$ prior line of therapy (39.3\%) than in patients who received $\geq 2$ lines (17.9\%). Disease control was achieved in $83.6 \%$ of cases. The response to treatment lasted for 5.4 months on average. The median progression-free survival was 6.8 months. The median overall survival was 12.5 months. The most frequent any-grade adverse events were hyperphosphatemia (73.2\%), fatigue $(49.3 \%)$, stomatitis $(45.1 \%)$, alopecia (38.0\%), and constipation (35.2\%). Grade 3-4 toxicity developed in 47 patients (66.2\%), including hypophosphatemia (14.1\%), hyperphosphatemia (12.7\%), and hyponatremia (11.3\%). Based on these results, the FDA approved an accelerated phase 3 clinical trial (PROOF 301 ), in which infigratinib is investigated as the firstline treatment for FGFR2-positive cholangiocarcinoma [22].

Therefore, FGFR2 monotherapy was a 2-3 times more effective therapeutic option with lower toxicity than old-fashion chemotherapy. Moreover, molecular agents have been associated with a higher response rate and prolonged duration of responses. These compounds have opened up an efficient second line of cholangiocarcinoma therapy that was not previously known. There is no doubt that it is necessary to assess the FGFR fusions and rearrangements in routine practice and, if present, to prescribe an FGFR inhibitor. There are a number of other FGFR inhibitors in phase 1 and $1 / 2$ trials, including futibatinib, derazantinib, Debio 1347, and erdafitinib. It can be assumed that FGFR inhibitors will be included in the standards of the first line of therapy in the near future.

Gastrointest Tumors 2021;8:153-158

DOI: $10.1159 / 000517258$ 


\section{IDH Inhibition}

Inhibition of IDH is another direction of targeted therapy for metastatic cholangiocarcinoma. An IDH mutation is found in intrahepatic cholangiocarcinoma cells in $10-20 \%$ of cases $[23,24]$. IDH exists in 3 isoforms, of which IDH1 and IDH2 play the greatest role in carcinogenesis. IDH1 mutations are more common than IDH2 mutations. Somatic IDH1/2 mutations appear in the early stages of tumor development. Increased IDH1/2 activity results in changes in the cellular metabolism and subsequent accumulation of the metabolite 2-hydroxyglutarate, which suppresses cell differentiation and induces tumorigenesis, in both tumor cells and blood. IDH1/2 mutations are not of prognostic interest; however, they are an excellent target for inhibition.

Ivosidenib, a small molecule inhibitor, inhibits IDH1 in cholangiocarcinoma cells harboring this mutation. Its efficacy has been studied in an international, multicenter, randomized phase 3 trial (ClarIDHy) [25]. Two hundred thirty patients with IHD1-positive metastatic cholangiocarcinoma resistant to standard chemotherapy were assigned in a 2:1 ratio to the ivosidenib (500 $\mathrm{mg}$, orally, daily, 28-day cycle; $n=124)$ or placebo group $(n=61)$. Patients could crossover from placebo to ivosidenib. The primary efficacy endpoint was progression-free survival.

With a median follow-up of 6.9 months, patients treated with ivosidenib had a significantly better progressionfree survival than patients treated with placebo $(\mathrm{HR}=$ $0.37 ; p<0.0001$ ). In absolute terms, the median was 2.7 and 1.4 months for this endpoint. The median overall survival was 10.8 months in the ivosidenib group and 9.7 months in the placebo group ( $\mathrm{HR}=0.69 ; p=0.06)$. The 6 -month and 1-year survival rates were $67 / 59 \%$ and $59 / 38 \%$, respectively. The favorable overall survival trend became statistically significant after adjusting for the $70 \%$ of patients crossing over to ivosidenib after radiographic disease progression [26]. In that adjusted analysis, risk was reduced by $51 \%(p<0.0001)$, and the median overall survival was 10.3 months with ivosidenib compared with 5.1 months with placebo. Two percent of patients achieved response to the inhibitor; there were no objective responses in the placebo group. Ivosidenib showed similar results in a phase 1 extension study $(n=73)$ with the objective response rate of $5 \%$ and the median progressionfree survival of 3.8 months [27]. As regards the reported toxicity in the ClarIDHy study, ascites (7\%) was the most common grade $\geq 3$ adverse event in both groups. Serious toxicity was reported in $30 \%$ of ivosidenib-treated patients and $22 \%$ of placebo-treated patients. There were no treatment-related deaths. Toxicity leading to treatment discontinuation was more common with placebo (8.5\%) than with ivosidenib (6.6\%). Health-related quality of life was also improved.

Again, targeted therapy is showing excellent results with a decrease in toxicity. The IDH1 inhibitors reduced the risk of disease progression by $63 \%$ and improved the overall survival by 2 times in pretreated patients. Data on progression-free survival and overall survival, combined with an acceptable safety profile and supportive healthrelated quality of life data, demonstrate the clinical benefit of ivosidenib in this aggressive disease for which there is an unmet need for new therapies.

\section{PD-1/PD-L1 Inhibition}

The immune system plays an important role in the pathogenesis of biliary tract tumors [28]. Several studies have shown that PD-L1 expression occurs in half of patients and correlates with a poor prognosis [29]. Immune checkpoint inhibitors have been studied in early clinical trials in patients with biliary tumors. In a multicenter phase 1 study, Japanese patients with biliary tract adenocarcinoma (intrahepatic bile duct cancer, extrahepatic bile duct cancer, gallbladder cancer, or ampullary cancer) and resistance to standard gemcitabine-based treatment regimens received nivolumab monotherapy [30]. A combination of nivolumab with gemcitabine and cisplatin was administered in chemotherapy-naïve patients. In the monotherapy group, the median progression-free survival was 1.4 months and the median overall survival was 5.2 months. In the combination therapy group, the median progression-free survival was 4.2 months and the median overall survival was 15.4 months. One of 30 patients and 11 of 30 patients achieved objective response in monotherapy and combination groups, respectively. The authors concluded that this trial provides supportive evidence for future larger studies of nivolumab in this difficult-to-treat cancer. A phase 2 study evaluated the efficacy and safety of the nivolumab monotherapy in patients with disease progression after 1-3 treatment lines [31]. The primary endpoint was the investigator-assessed objective response rate of $22 \%(10$ of 46 patients), with a disease control rate of $59 \%$ (27 of 46 patients) and durable responses over 1 year. The median progression-free survival was 3.68 months and the median overall survival was 14.24 months. Both trials demonstrated better outcomes in patients with PD-L1 expression. 
Another checkpoint inhibitor, pembrolizumab, has been studied in phase $1 \mathrm{~b}$ and phase 2 basket trials [32]. A phase $1 \mathrm{~b}$ study (KEYNOTE 028) included 24 PD-L1-positive (PD-L1 $\geq 1 \%$ ) patients and reported an objective response rate of $13 \%$. The median progression-free survival and the median overall survival were 1.8 and 6.2 months, respectively. The one-year survival rate was $27.6 \%$. In the phase 2 study (KEYNOTE 158), 61 of 104 patients had PD-L1 expression. Despite a 2 -fold decrease in the response rate $(5.8 \%)$ compared to the previous study, the median progression-free survival (2 months), median overall survival (7.4 months), and 1-year survival rate $(32.7 \%)$ were very similar. Bang et al. [32] concluded that pembrolizumab provided durable antitumor activity, regardless of PD-L1 expression, and manageable toxicity.

Durvalumab alone $(n=42)$ or in combination with tremelimumab $(n=65)$ was also investigated in Asian patients with advanced biliary tumors and disease progression on previous systemic therapy [33]. In the durvalumab group, 2 patients had partial responses, and 7 patients had partial responses in the combination group. The disease control rate at 12 weeks was 16.7 and $32.2 \%$, respectively. The median duration of response for the durvalumab group was 9.7 months and 8.5 months for the combination group. The median overall survival was 8.1 and 10.1 months in these groups.

Finally, a randomized, open-label, multicenter phase 2 trial $(n=77)$ showed activity of atezolizumab (anti-PDL1 inhibitor) in combination with cobimetinib (MEK inhibitor) in patients with 1-2 lines of prior therapy for biliary tract cancers including cholangiocarcinoma [34]. The trial met its primary endpoint, with a median progression-free survival of 3.65 months and $45.1 \%$ of objective responses. Overall survival data were not mature at the time of analysis. Immunotherapy has given hope to patients with cholangiocarcinoma. Preliminary results indicate that this treatment can be successful in this cohort of patients. A phase 3 study is required in which checkpoint inhibitors should be compared with standard chemotherapy.

\section{Conclusions}

We are witnessing a revolution in the treatment of cholangiocarcinoma today. The treatment-refractory tumor has begun to lose ground amid treatment with FGFR inhibitors and IDH inhibitors. A total of $25 \%$ of patients have molecular genetic alterations that allow considering such therapy. FGFR2 inhibition drastically increased objective response rates, progression-free survival, and overall survival compared with chemotherapy in previously treated patients, serving as the basis for FDA approval of pemigatinib for the second-and subsequent-line therapy. Despite the fact that the results with IDH inhibition seem to be inferior to those with FGFR inhibition, this treatment option is also viable. First, a significant difference in the primary endpoint was achieved in the study, and the risk of disease progression was reduced by $63 \%$; second, patients who have relapsed after prior lines of therapy have no other options; third, IDH-mutant cholangiocarcinoma is a separate tumor type that requires a special approach. Based on the effects of the IDH1 mutation on tumors, for instance, a role in DNA repair, one possibility is to combine IDH1 inhibitors with inhibitors of poly (ADPribose) polymerase.

Promising clinical benefit was also observed with immune checkpoint therapy. Apparently, the assessment of PD-L1 expression will not be necessary for the treatment initiation. Nivolumab, pembrolizumab, durvalumab, and tremelimumab showed intriguing results in heavily pretreated patients. Another possible option, based on modulation of the immune microenvironment, is to combine FGFR or IDH1 inhibitors with checkpoint inhibitors. Looking into the future, we can expect the emergence of these drugs in the first-line therapy, as well as investigation of new targets for targeted molecular therapy. At the Hadassah Institute of Oncology, we are conducting a registry study that will help assess the impact of new treatments in routine practice.

\section{Conflict of Interest Statement}

The authors have no conflicts of interest to declare.

\section{Funding Sources}

The authors did not receive any funding.

\section{Author Contributions}

All the authors: drafting of the manuscript and final approval of the manuscript.
Targeted Therapy and Immunotherapy

for Cholangiocarcinoma
Gastrointest Tumors 2021;8:153-158 DOI: $10.1159 / 000517258$ 


\section{References}

1 Breder VV, Bazin IS, Kosyrev VY, Ledin EV. Practical recommendations for drug treatment of bile duct cancer. In: Malignant tumors: RUSSCO practice guidelines \# 3s2; 2019. Vol. 9; p. 439-55.

2 Patel N, Benipal B. Incidence of cholangiocarcinoma in the USA from 2001 to 2015: a US cancer statistics analysis of 50 states. Cureus. 2019;11(1):e3962.

3 Khan SA, Davidson BR, Goldin RD, Heaton N, Karani J, Pereira SP, et al. Guidelines for the diagnosis and treatment of cholangiocarcinoma: an update. Gut. 2012;61:1657-69.

4 Yamamoto M, Takasaki K, Otsubo T, Katsuragawa H, Katagiri S. Recurrence after surgical resection of intrahepatic cholangiocarcinoma. J Hepatobiliary Pancreat Surg. 2001;8:154-7.

5 Valle J, Wasan H, Palmer DH, Cunningham D, Anthoney A, Maraveyas A, et al. Cisplatin plus gemcitabine versus gemcitabine for biliary tract cancer. N Engl J Med. 2010;362(14):1273-81.

6 Lamarca A, Palmer DH, Singh Wasan H, Ross PJ, Ma YT, Arora A, et al. ABC-06|A randomised phase III, multi-centre, open-label study of active symptom control (ASC) alone or ASC with oxaliplatin / 5-FU chemotherapy (ASC+mFOLFOX) for patients (pts) with locally advanced / metastatic biliary tract cancers $(\mathrm{ABC})$ previously-treated with cisplatin/gemcitabine (CisGem) chemotherapy. Proc Am Soc Clin Oncol. 2019;37(Suppl 15):4003. (abstr).

7 Lowery MA, Goff LW, Keenan BP, Jordan E, Wang R, Bocobo AG, et al. Second-line chemotherapy in advanced biliary cancers: a retrospective, multicenter analysis of outcomes. Cancer. 2019;125:4426-34.

8 Ying J, Chen J. Combination versus monotherapy as salvage treatment for advanced biliary tract cancer: a comprehensive meta-analysis of published data. Crit Rev Oncol Hematol. 2019;139:134-42.

9 Valle JW, Lamarca A, Goyal L, Barriuso J, Zhu AX. New horizons for precision medicine in biliary tract cancers. Cancer Discov. 2017;7: 943-62.

10 Nakazawa K, Dobashi Y, Suzuki S, Fujii H, Takeda Y, Ooi A. Amplification and overexpression of c-erbB-2, epidermal growth factor receptor, and c-met in biliary tract cancers. J Pathol. 2005;206:356-65.

11 Terada T, Nakanuma Y, Sirica AE. Immunohistochemical demonstration of MET overexpression in human intrahepatic cholangiocarcinoma and in hepatolithiasis. Hum Pathol. 1998;29:175-80.

12 Goyal L, Zheng H, Yurgelun MB, Abrams TA, Allen JN, Cleary JM, et al. A phase 2 and biomarker study of cabozantinib in patients with advanced cholangiocarcinoma. Cancer. 2017 Jun 1;123(11):1979-88.

13 Helsten T, Elkin S, Arthur E, Tomson BN, Carter J, Kurzrock R. The FGFR landscape in cancer: analysis of 4,853 tumors by next-generation sequencing. Clin Cancer Res. 2016;22: 259-67.
14 Tsimafeyeu I, Ludes-Meyers J, Stepanova E, Daeyaert F, Khochenkov D, Joose J-B, et al. Targeting FGFR2 with alofanib (RPT835) shows potent activity in tumour models [published correction appears. Eur J Cancer. 2016; 61:20-8.

15 Statsenko G, Fedyanin M, Moiseyenko V, Vladimirova LY, Tsimafeyeu I, Tjulandin S. A phase Ib study of alofanib, an allosteric FGFR2 inhibitor, in patients with advanced or metastatic gastric cancer. J Clin Oncol. 2020; 38(4_Suppl 1):TPS466.

16 Graham RP, Barr Fritcher EG, Pestova E, Schulz J, Sitailo LA, Vasmatzis G, et al. Fibroblast growth factor receptor 2 translocations in intrahepatic cholangiocarcinoma. Hum Pathol. 2014;45:1630-8.

17 Abou-Alfa GK, Sahai V, Hollebecque A, Vaccaro G, Melisi D, Al-Rajabi R, et al. Pemigatinib for previously treated, locally advanced or metastatic cholangiocarcinoma: a multicentre, open-label, phase 2 study. Lancet Oncol. 2020;21(5):671-84.

18 FDA. Available from: https://www.fda.gov/ drugs/resources-information-approveddrugs/fda-grants-accelerated-approval-pemigatinib-cholangiocarcinoma-fgfr2-rearrangement-or-fusion.

19 Abou-Alfa GK, Sahai V, Hollebecque A, Vaccaro G, Melisi D, Al-Rajabi R, et al. Pemigatinib for previously treated, locally advanced or metastatic cholangiocarcinoma: a multicentre, open-label, phase 2 study. Lancet Oncol. 2020;21(5):671-84.

20 Bekaii-Saab TS, Valle JW, Cutsem EV, Rimassa L, Furuse J, Ioka T, et al. FIGHT-302: firstline pemigatinib vs. gemcitabine plus cisplatin for advanced cholangiocarcinoma with FGFR2 rearrangements. Future Oncol. 2020; 16(30):2385-99.

21 Javle M, Kelley RK, Roychowdhury S, Weiss $\mathrm{KH}$, Abou-Alfa GK, Macarulla T, et al. Updated results from a phase II study of infigratinib (BGJ398), a selective pan-FGFR kinase inhibitor, in patients with previously treated advanced cholangiocarcinoma containing FGFR2 fusions. Ann Oncol. 2018;29(8): viii720.

22 Makawita S, Abou-Alfa GK, Roychowdhury S, Sadeghi S, Borbath I, Goyal L, et al. Infigratinib in patients with advanced cholangiocarcinoma with FGFR2 gene fusions/translocations: the PROOF 301 trial. Future Oncol. 2020;16(30):2375-84.

23 Boscoe AN, Rolland C, Kelley RK. Frequency and prognostic significance of isocitrate dehydrogenase 1 mutations in cholangiocarcinoma: a systematic literature review. J Gastrointest Oncol. 2019;10:751-65.

24 Dang L, Jin S, Su SM. IDH mutations in glio$\mathrm{ma}$ and acute myeloid leukemia. Trends Mol Med. 2010;16:387-97.

25 Abou-Alfa GK, Macarulla T, Javle MM, Kelley RK, Lubner SJ, Adeva J, et al. Ivosidenib in IDH1-mutant, chemotherapy-refractory chol- angiocarcinoma (ClarIDHy): a multicentre, randomised, double-blind, placebo-controlled, phase 3 study. Lancet Oncol. 2020;21:796-807.

26 Zhu AX, Macarulla T, Javle MM, Kelley RK, Lubner SJ, Adeva J, et al. Final results from ClarIDHy, a global, phase III, randomized, double-blind study of ivosidenib versus placebo in patients with previously treated cholangiocarcinoma and an isocitrate dehydrogenase 1 (IDH1) mutation. Gastrointestinal Cancers Symposium; Presented 17 Jan 2021; 2012. Abstract 266.

27 Lowery MA, Burris HA 3rd, Janku F, Shroff RT, Cleary JM, Azad NS, et al. Safety and activity of ivosidenib in patients with IDH1mutant advanced cholangiocarcinoma: a phase 1 study. Lancet Gastroenterol Hepatol. 2019;4:711-20.

28 Asahi Y, Hatanaka KC, Hatanaka Y, Kamiyama T, Orimo T, Shimada S, et al. Prognostic impact of CD8+ T cell distribution and its association with the HLA class I expression in intrahepatic cholangiocarcinoma. Surg Today. 2020;50(8):931-40.

29 Xie Q, Wang L, Zheng S. Prognostic and clinicopathological significance of PD-L1 in patients with cholangiocarcinoma: a meta-analysis. Dis Markers. 2020;2020:1817931.

30 Ueno M, Ikeda M, Morizane C, Kobayashi S, Ohno I, Kondo S, et al. Nivolumab alone or in combination with cisplatin plus gemcitabine in Japanese patients with unresectable or recurrent biliary tract cancer: a non-randomised, multicentre, open-label, phase 1 study. Lancet Gastroenterol Hepatol. 2019; 4(8):611-21.

31 Kim RD, Chung V, Alese OB, El-Rayes BF, Li $\mathrm{D}, \mathrm{Al}$-Toubah TE, et al. A Phase 2 multi-institutional Study of nivolumab for patients with advanced refractory biliary tract cancer. JAMA Oncol. 2020;6(6):888-94.

32 Bang Y-J, Ueno M, Malka D, Chung HC, Nagrial A, Kelley RK, et al. Pembrolizumab (pembro) for advanced biliary adenocarcinoma: results from the KEYNOTE-028 (KN028) and KEYNOTE-158 (KN158) basket studies. J Clin Oncol. 2019;37(15_Suppl 1):4079.

33 Ioka T, Ueno M, Oh D-Y, Fujiwara Y, Chen J-S, Doki Y, et al. Evaluation of safety and tolerability of durvalumab (D) with or without tremelimumab (T) in patients (pts) with biliary tract cancer (BTC). J Clin Oncol. 2019; 37(4_Suppl 1):387.

34 Yarchoan M, Cope L, Anders RA, Noonan A, Goff LW, Goyal L, et al. A multicenter randomized phase 2 trial of atezolizumab as monotherapy or in combination with cobimetinib in biliary tract cancers (BTCs): a NCI experimental therapeutics clinical trials network (ETCTN) study [abstract]. Proceedings of the Annual Meeting of the American Association for Cancer Research 2020; $2020 \mathrm{Apr}$ 27-28 and Jun 22-24; Philadelphia, PA: AACR; 2020. Vol. 80(16 Suppl). Abstract nr CT043. 